\title{
BMJ Open Comprehensive Cervical Cancer Prevention in Tanzania (CONCEPT) study: Cohort profile
}

\author{
Bariki Mchome, ${ }^{1}$ Patricia Swai, ${ }^{1}$ Chunsen Wu, ${ }^{2,3}$ Johnson Katanga, ${ }^{4}$ \\ Crispin Kahesa, ${ }^{4}$ Rachel Manongi, ${ }^{5}$ Julius D Mwaiselage, ${ }^{4}$ Susanne Kjaer, ${ }^{6,7}$ \\ Vibeke Rasch, ${ }^{2,3}$ Ditte Søndergaard Linde (1) ${ }^{2,3}$
}

To cite: Mchome B, Swai P, Wu C, et al. Comprehensive Cervical Cancer Prevention in Tanzania (CONCEPT) study: Cohort profile. BMJ Open 2020;10:e038531. doi:10.1136/ bmjopen-2020-038531

- Prepublication history and additional material for this paper are available online. To view these files, please visit the journal online (http://dx.doi. org/10.1136/bmjopen-2020038531).

Received 13 March 2020 Revised 13 July 2020 Accepted 16 July 2020
Check for updates

(C) Author(s) (or their employer(s)) 2020. Re-use permitted under CC BY-NC. No commercial re-use. See rights and permissions. Published by BMJ.

For numbered affiliations see end of article.

Correspondence to Ditte Søndergaard Linde; dsondergaard@health.sdu.dk

\section{ABSTRACT}

Purpose Cervical cancer is a major cause of death among women in Eastern Africa, and the distribution of human papillomavirus (HPV) according to HIV status is inadequately characterised in this region. In order to guide future cervical cancer preventive strategies that involve HPV testing, the Comprehensive Cervical Cancer Prevention in Tanzania (CONCEPT) study was established in 2015. The CONCEPT cohort aims to investigate the natural history of HPV and determine acquisition and persistence patterns of high-risk (HR) HPV among HIV-positive and HIV-negative women. Further, the influence of lifestyle and sexual/reproductive factors will be investigated. The main objective of this article is to describe how the CONCEPT cohort was established.

Participants Women aged 25-60 years were enrolled from cervical cancer screening clinics in Dar-es-Salaam and Moshi, Tanzania. Data were collected at baseline, at 14 months (first follow-up) and at 28 months (second follow-up). Biological samples included two cervical swabs for careHPV DNA testing, cytology, Hybrid Capture 2, genotyping and blood samples for HIV. Visual inspection with acetic acid was performed, and sociodemographic, lifestyle and sexual/reproductive characteristics were collected through a standardised questionnaire.

Findings to date 4043 women were included in the cohort from August 2015 to May 2017. At baseline, 696 (17.1\%) women were HR HPV positive, and among these, $31.6 \%$ were HIV positive; 139 women (3.4\%) had highgrade squamous intraepithelial lesions. 3074 women $(81 \%)$ attended the first follow-up. The majority attended after receiving a phone call reminder $(35 \%)$ or from home via self-samples (41\%). At first follow-up, 438 (14.4\%) were HR HPV positive and $30.4 \%$ of these were HIV positive.

Future plans A second follow-up is underway (17 December 2018-0ctober 2020). We plan to integrate our data with a previous cross-sectional HPV study from Tanzania to increase the power of our findings. Researchers interested in collaborating are welcomed, either by extracting data or jointly requesting further investigation from the cohort.

\section{INTRODUCTION}

Cervical cancer is a major cause of cancerrelated mortality and morbidity globally.

\section{Strengths and limitations of this study}

- This is a large-scale longitudinal cervical cancer study conducted in an HIV endemic population that aims to address a major cause of disease among East-African women, which so far has not received much focus within global health research.

- Women are followed up over a long duration of time and with a large amount of data being collected by using questionnaires and laboratory tests.

- It was difficult to get women to return for followup screenings. However, carefully designed tracing plans and dedicated home-visiting staff entailed that we managed to attain an $81 \%$ participation rate at first follow-up.

Detailed HIV documentation was challenging to obtain, which has limited the power in analyses involving HIV immunologic markers and treatment.

The highest prevalence is found among women aged 45-60years ${ }^{1}$, and the burden of disease is disproportionally distributed among low-income and middle-income countries (LMICs) and high-income countries (HICs)-LMICs account for $80 \%$ of cervical cancer cases worldwide. The global age-standardised incidence rate for cervical cancer is 14 per 100000 women, ${ }^{2}$ while the incidence rate of cervical cancer is 42.7 per 1000000 women in East Africa ${ }^{3}$ and 54 per 100000 women in Tanzania, specifically. ${ }^{4}$ Major contributing factors to the high burden of disease in resource-limited settings includes low awareness of the disease and how to prevent it and unavailability of organised screening programmes. The standard screening test in resource-limited settings is visual inspection with acetic acid (VIA) as this can be performed by mid-level providers and allows for immediate treatment. However, the result of VIA is a subjective interpretation resulting in variable performances, and the utility is questionable in resource-limited 
settings when the number of screening rounds per women's lifetime is low. ${ }^{5}$

Human papillomavirus (HPV) is the most common sexually transmitted infection worldwide, and there is a $60 \%-70 \%$ lifetime risk of acquiring an HPV infection among sexually active women. ${ }^{6}$ Eighty per cent to $90 \%$ of HPV infections clear spontaneously; however, 10\%-20\% become persistent and can develop into precancerous lesions and cervical cancer over time. There are different factors associated with HPV persistence, the two most significant ones are the type of HPV involved and immunodeficiency, hence HIV-positive women have increased risk of acquiring $\mathrm{HPV}^{7}$ and for the infection to become persistent. ${ }^{89}$ HPV16 and 18 are the two most important types as these are associated with approximately $70 \%$ of all invasive cervical cancers worldwide. ${ }^{6}$ Globally, the five most common types are HPV16, 18, 52, 31 and $58 .{ }^{10} 11$ However, cross-sectional studies from Africa and systematic reviews on the global HPV burden indicate that the type-specific prevalence of HPV differs in Africa compared with other regions, ${ }^{2} 1213$ specifically HPV 52, 58, 31 and 35 are more common in African countries compared with other parts of the world. ${ }^{14-16}$ Tanzanian data from the HPV information centre have found the most prevalent HR HPV types among Tanzanian women with high-grade squamous intraepithelial lesions (HSILs) to be HPV 16 (30.2\%), HPV 52 (21.9\%) and HPV 18 $(16.7 \%)$, while the most prevalent HPV types among women with cervical cancer are HPV 16 (47.7\%), HPV 18 (18.2\%) and HPV 45 (11.4\%). ${ }^{17}$ Further, sexual, reproductive and lifestyle factors influence HPV acquisition and persistence, including smoking, high parity, number of sexual partners, long-term use of oral contraceptives and coinfections with other sexually transmitted agents. ${ }^{18} 19$ However to date, there are no adequately powered longitudinal HPV studies among middle-aged women in East Africa that explore the association of HIV, immunological factors, reproductive and lifestyle factors on HR HPV acquisition and persistence.

To our knowledge, only two HPV cohort studies and one large HPV cross-sectional study have been conducted in Africa, which explore the dynamics of HPV, HIV and cervical cancer, namely (1) the HPV in Africa Research Partnership (HARP) HPV Study in Burkina Faso and Tanzania $^{20}$; (2) the African Collaborative Center for Microbiome and Genomics Research (ACCME) study in Nigeria $^{21}$; and (3) the Prevention of Cervical Cancer in Tanzania (PROTECT) study. ${ }^{22}$ Other studies are nested in HPV vaccine trials. ${ }^{23-25}$ These studies have provided some insight into the distribution of HPV among different African populations; however, they were either cross sectional or conducted among adolescents with inadequately powered HIV-positive women and with a relatively short duration of follow-up.

The Comprehensive Cervical Cancer Prevention in Tanzania (CONCEPT) study was launched in 2015 with an overall aim of improving prevention of cervical cancer in Tanzania (online supplementary appendix 1). The
CONCEPT study is an international collaborative study between Ocean Road Cancer Institute (ORCI), Kilimanjaro Christian Medical Centre (KCMC), University of Southern Denmark, and the Danish Cancer Society Research Center. The CONCEPT study has several specific objectives: (1) to investigate the natural history of HPV and its associated factors; (2) to determine the feasibility and acceptability of HPV self-sampling ${ }^{26}$ and the test performance of careHPV compared with (HC2) and $\mathrm{VIA}^{11}$; and (3) how to ensure follow-up of HPV-positive women (ClinicalTrials.gov NCT02509702), and elucidate what motivates or prevents these women from attending to follow-up visits. ${ }^{27-29}$ Introduction of HPV DNA testing in LMICs is challenging due to logistical problems inherent in these settings; however, HPV-based primary screening is a key method in future screening programmes across the world, ${ }^{26}$ and for it to be effectively established in resourcelimited settings, local-specific evidence is warranted. The aim of this article is to describe how this cohort was established and followed up, the profile of the cohort, and provide some characteristics of the cohort at enrolment and at the first follow-up. The specific objectives of the CONCEPT study have been and will be published in separate papers. $^{112830-33}$

\section{COHORT DESCRIPTION}

\section{Study design and study population}

This study was conducted in Tanzania, which is a lowincome country located in Eastern Africa with a population of 56 million people. ${ }^{10}$ Women were enrolled from three existing cervical cancer screening clinics located in urban and semirural areas: (1) ORCI in Dar-es-Salaam as well as (2) KCMC and (3) Mawenzi regional referral hospital in the Kilimanjaro region. ORCI is a national cancer hospital that provides clinical care and treatment for all patients with cancer in the country. Additionally, they conduct cervical cancer screening three times a week for the general population. KCMC is a northern zonal tertiary facility that provides cervical screening three times a week for the general population, and Mawenzi Hospital is a regional hospital that provides cervical cancer screening two times a week. In Dar-es-Salaam, women from Ilala, Temeke and Mwananyamala district were included, while in the Kilimanjaro region, women originating from the urban and rural district of Moshiincluding Hai and Rombo-were included. Originally, the study was designed as a double-site study (KCMC/ORCI), however, due to a slower-than-anticipated recruitment rate, a third study site (Mawenzi) was added 6 months into the enrolment period. Women were eligible for inclusion if they were 25-60 years and attended a patient-initiated routine cervical cancer screening at one of the study sites. Women were excluded if they were pregnant, on their menstrual period, had a history of premalignant lesions of the cervix within the last 12 months, had previously been diagnosed with cervical cancer or had undergone abdominal hysterectomy. Women on their menstrual 
period were encouraged to return once their menstrual period was over. Following a detailed explanation of the study, all participants provided written informed consent. Fingerprints were used for illiterate participants. HIV positive women were oversampled from care and treatment clinics (CTC) at the study sites from where they were referred to the screening clinics. The total number of women and HIV positives required for the study was found through a power calculation based on McNemar's test comparing two diagnostic tests (S1: standard test (VIA) vs S2: new test (careHPV) with cervical cytology as the reference test and the threshold being HSIL+ (HSIL, carcinoma in situ and carcinoma). The power calculation was based on the research group's previous study in Tanzania. ${ }^{22}$ It was estimated that $180-200$ women would have precancerous lesions at baseline ( true positives), and assuming a significance level of $5 \%, 80 \%$ power and a sensitivity of VIA of $30 \%$, it would be possible to detect a significant difference if the sensitivity of the new test would be at least $44 \%$. It was anticipated that careHPV testing would have a higher sensitivity than VIA.

Data were collected during the enrolment visit, at 14 months (first follow-up) and at 28 months (second follow-up, ongoing). As there is no predefined optimal duration of time to investigate the natural history of HPV, the length of follow-up was based on a number of factors, including the recommended duration of time between cervical cancer screenings for HIV positive women (12 months), ${ }^{34}$ available resources, risk for developing cervical lesions and limiting the workload at the screening clinics by minimising overlaps between enrolment and follow-up visits. Healthcare providers working at the screening clinics enrolled participants and collected data following protocols developed specifically for the project. At inclusion, all participants were given a 14-month follow-up appointment written on an appointment card. If the women did not attend their follow-up visit within 1 month of their appointment, an active follow-up procedure was initiated. First, non-attendees were contacted by phone and encouraged to attend (tracing method I). If the woman did not show up within 2 weeks of the phone call, the women had a home-visit by an outreach nurse who encouraged her to attend (tracing method II). If the woman still did not attend and consented to it, an outreach nurse visited her again and conducted the follow-up visit at home (tracing method III). Transportation costs were compensated for those women who were reminded to come. Women who participated in the first follow-up were appointed a second follow-up visit 28 months after initial recruitment. If the woman did not attend the second follow-up, tracing method I and III were reinitiated.

\section{Assessment of exposure}

At inclusion, sociodemographic, lifestyle, reproductive and sexual characteristics were collected during a personal interview using a modified version of a standardised questionnaire adopted from a previous study conducted in Tanzania ${ }^{35}$ (online supplementary appendix 3). The questionnaire was hard copy, developed in English while the interview was conducted in Kiswahili with direct translation. A Kiswahili version of the questionnaire was available to guide the interviewers. A detailed contact information form was filled at enrolment and updated at follow-up. Participants were screened by trained healthcare providers according to the standard national cervical cancer screening prevention programme in Tanzania. ${ }^{36}$ This entails a cost-free gynaecological examination with VIA, HIV testing and HIV counselling. Venous blood from the index finger was tested by using a quick HIV-1/2 test ( www.alere.com), and a supplementary quick HIV-1/2 test (Abbott Laboratories) was performed as confirmatory for positive results. Discordant results were further confirmed using Unigold (Trinity Biotech). Women who screened VIA positive or were suspected of manifest cancer were managed on-site based on the National Cervical Cancer Service Delivery Guidelines. This included treatment with cryotherapy or loop electrosurgical excision procedure for VIA positives, and punch biopsy and referral for treatment at the oncology clinic at ORCI for cancer suspicions. ${ }^{36}$ Further, weight and height were measured and registered on a hard-copy registration sheet together with the HIV and VIA result (table 1).

Prior to the routine VIA examination, cervical swabs were taken using (1) an Aryes spatula for careHPV test (www.qiagen.com), and another specimen was taken using (2) a ThinPrep Pap test plastic spatula for liquidbased cytology, HPV DNA testing and genotyping by use of HC2 and LiPaExtra (Innogenetics, Gent, Belgium). The cervical samples for careHPV analysis were kept in a careHPV collection medium and stored at room temperature $\left(\max 25^{\circ} \mathrm{C}\right)$ in laboratories at ORCI or KCMC. Once 90 samples had been collected, they were analysed for HR HPV using a careHPV machine. A test was considered positive if one or more of the following $14 \mathrm{HR}$ HPV types were detected: HPV16, 18, 31, 33, 35, 39, 45, 51, 52, 56, $58,59,66,68$. The results were registered on a careHPV results sheet (table 1 ).

The samples for HC2 testing, genotyping and cytology were kept in a PreServCyt solution (Hologic, 250 Campus Drive, Marlborough, MA 01752, USA) and stored at room temperature in laboratories at ORCI and KCMC. Once enrolment had finished, the PreServCyt vials were sent to the Department of Pathology at Lillebaelt Hospital, Vejle, Denmark and processed at the ThinPrep5000 Autoloader Instrument, Hologic, according to manufacturer's instruction and stained with ThinPrep Stain. The slides were scanned by the Thin Prep Imaging System with selection of 22 fields of view which was reviewed by cytotechnologist in review scopes. The specimens were evaluated for adequacy and for abnormal cells. If abnormal cells were detected, the slides were consulted with a gynae-pathologist who made the final diagnosis. The specimens were diagnosed according to the Bethesda Nomenclature for System for Cervical Cytology $2014^{37}$ into following categories: negative for intraepithelial 


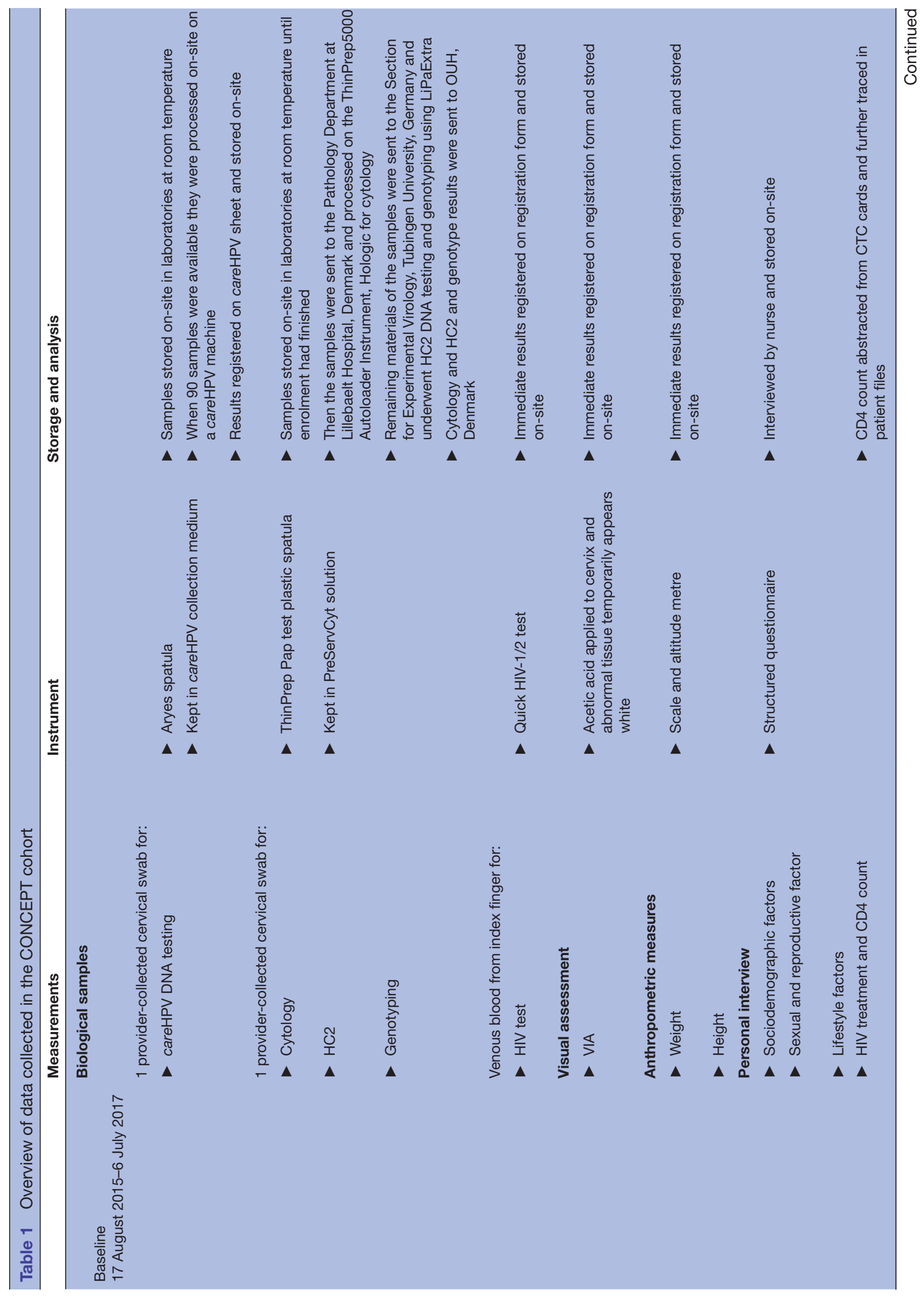




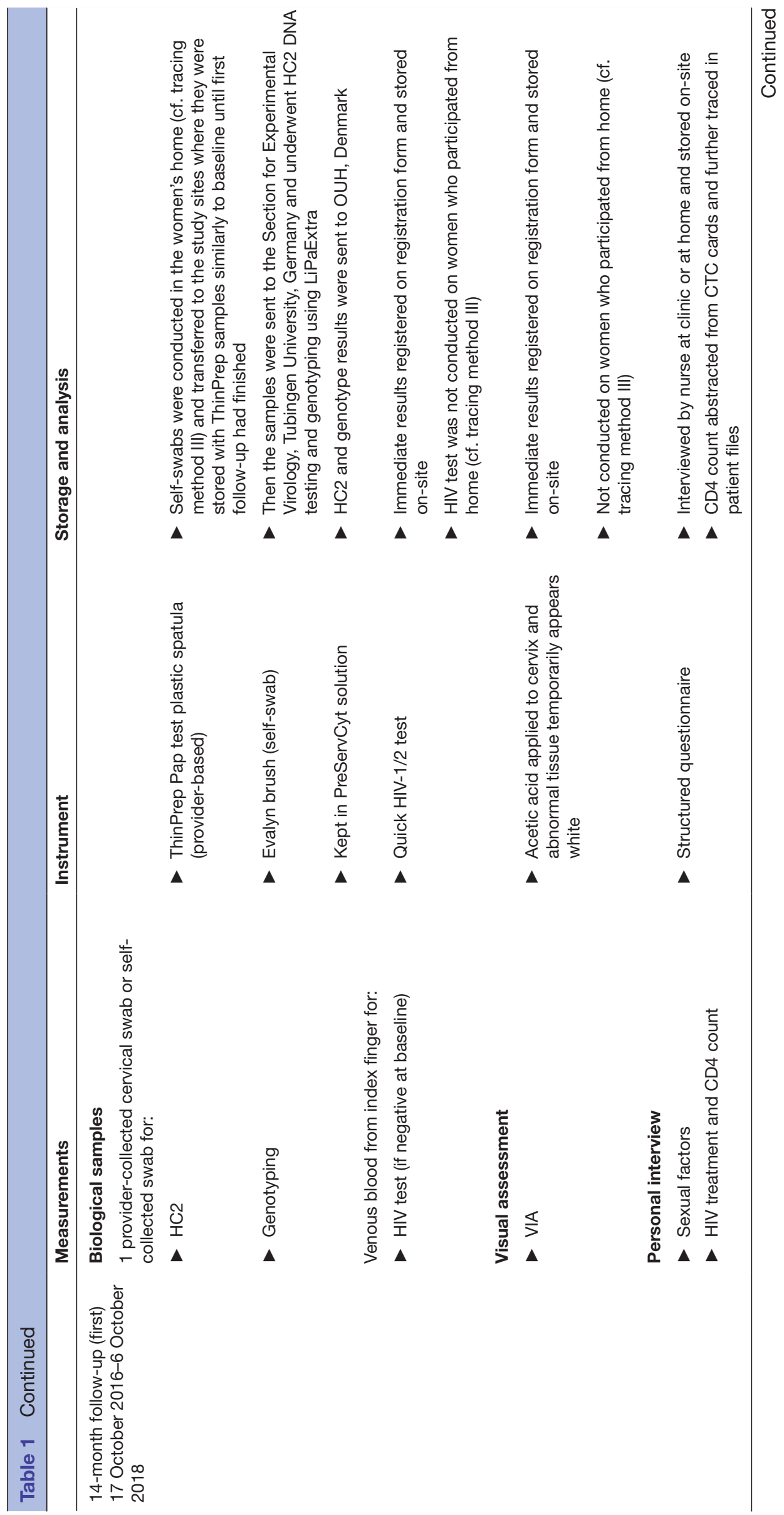




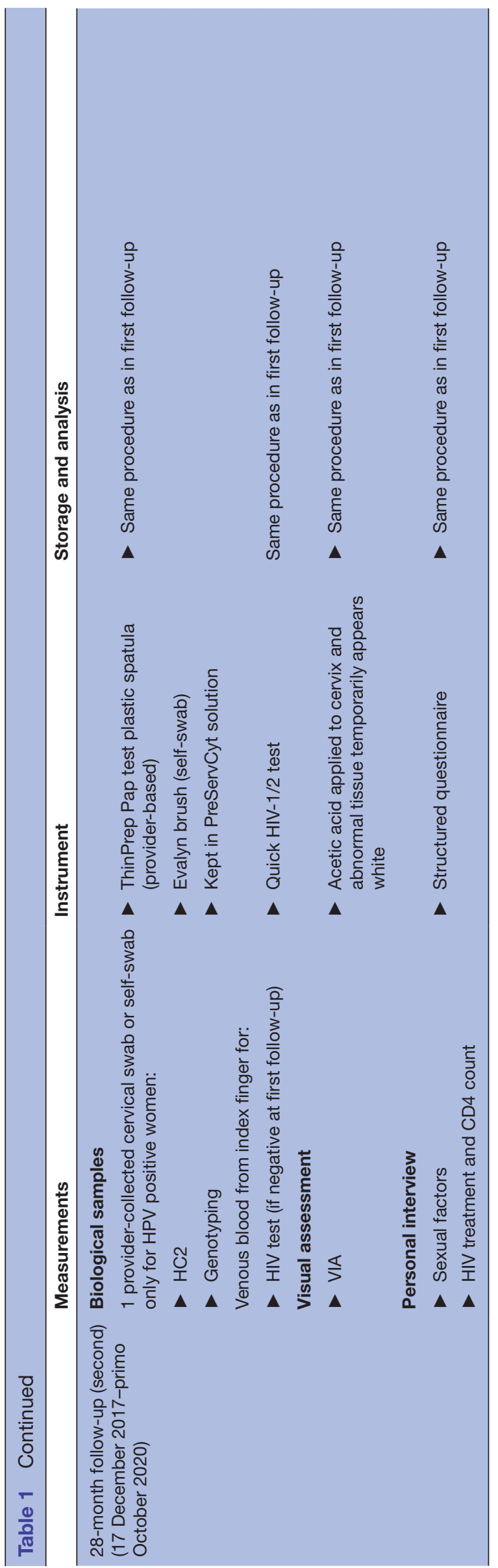

lesion (NILM), atypical squamous cell of undetermined significance (ASCUS), atypical squamous cell in which high-grade squamous intraepithelial lesion cannot be excluded (ASCH), low-grade squamous intraepithelial lesion (LSIL), high-grade intraepithelial lesion (HSIL), atypical glandular cell (AGC), adenocarcinoma in situ (AIS) and adenocarcinoma. The remaining material of the PreServCyt vials were sent to the Section for Experimental Virology, Tubingen University, Germany, for HPV DNA testing and genotyping. HPV DNA testing was done using HC2 DNA test (www.qiagen.com) with a HR cocktail probe. A test was considered positive if one or more of the following 14HR HPV types were found: HPV 16, 18, $31,33,35,39,45,51,52,56,58,59,66,68$. A threshold of $1.0 \mathrm{pg}$ HPV DNA $/ \mathrm{ml}$, which corresponds to 1.0 relative light unit coefficient, was used, as recommended by the US Food and Drug Authority. HPV positive samples were genotyped using LiPaExtra, which can detect $28 \mathrm{HPV}$ types, $18 \mathrm{HR}$ risk types (HPV 16, 18, 26, 31, 33, 35, 39, 45, $51,52,53,56,58,59,66,68,73,82)$ and 10 low risk types (HPV 6, 11, 40, 43, 44, 54, 69, 70, 71, 74). ${ }^{38}$

\section{Outcome measures}

Women who attended their follow-up appointment at the screening clinics were HIV tested (if negative at baseline), had a cervical swab collected with a ThinPrep Pap test plastic spatula-for HPV DNA testing using HC2 and genotyping using LiPaExtra-and underwent VIA (table 1). Further, sexual and reproductive characteristics were updated using a structured questionnaire (online supplementary appendix 4). Women who did not attend their follow-up appointment at the clinic but consented to having a home-visit appointment (cf. tracing method III) responded to the questionnaire and had cervical specimens collected by use of an Evalyn self-sampling/ self-swab device (www.roversmedicaldevices.com). The samples were transferred to laboratories at ORCI and KCMC where they were kept in a PreServCyt solution and stored at room temperature.

\section{Data management}

Questionnaires, registrations forms, contact forms and careHPV result sheets were stored in different cabinets in locked offices at KCMC and ORCI, after which they were double entered into EpiData by data clerks. Together with laboratory results, these data were sent to the Research Unit for Gynaecology \& Obstetrics, Odense University Hospital (OUH), Odense, Denmark, where they were merged and constructed into a baseline database. Data with invalid identification numbers (IDs) or no data registered were excluded on creation of the database. Similar to baseline, follow-up data were sent to OUH, after which it was merged with the baseline database. Follow-up IDs that could not match a baseline ID were excluded.

\section{Patient and public involvement}

Study participants were not involved in the design or recruitment of the study. In order to increase public 
awareness, government and religious leaders were informed about the project, the latter through mosques and churches. When the study finishes, the results and their potential implication to the public will be communicated through meetings with health authorities, policy briefings and announcements in the mainstream media.

\section{FINDINGS TO DATE \\ Baseline findings}

A total of 4080 women were enrolled into the CONCEPT study. After inclusion, 37 study participants were excluded, leaving the total cohort to consist of 4043 women (figure 1). The baseline sociodemographic distribution is presented in table 2. A total of 718 women $(17.8 \%)$ were HIV positive and the majority of these were $35-39$ years old (22.9\%) while the majority of HIV negative women were $40-44$ years old $(19.2 \%)$. Among the HIV positives, $49.7 \%$ were married $(n=356)$ while $73.6 \%$ of HIV negatives were married $(n=2434)$. Most of both HIV positive $(70.4 \%$; $=504)$ and HIV negative women $(64.1 \% ; \mathrm{n}=2127)$ had completed primary school. More HIV negative women $(87.0 \% ; n=2879)$ reported to have had sex within the last 12 months compared with HIV positive women $(72.7 \% ; \mathrm{n}=520)$, while more HIV positives $(26.2 \%)$ than HIV negatives $(9.8 \%)$ reported to have had used condoms at every intercourse. In relation to number of lifetime partners, $71.9 \%$ of HIV positives reported having more than one lifetime partner while the corresponding number for HIV negative women was $61.0 \%$. The CD4 count for HIV positive women were as follows: $8.6 \%(n=62)$ reported having a $\mathrm{CD} 4$ count $\leq 199$; $30.5 \%(\mathrm{n}=219)$ had a CD4 ranging from 200 to 499; and
$48.9 \%(\mathrm{n}=347)$ had a CD4 count $\geq 500$. Further, $12.5 \%$ $(\mathrm{n}=90)$ of the HIV positives did not report the CD4 count.

Among the 4043 participants, the cervical sample was insufficient for HPV analysis for 396 women (9.8\%) at baseline, leaving 3667 women eligible for analysis of HPV positivity. Further, 27 women $(0.5 \%)$ did not have a sample for cervical cytology, leaving 4016 women available for cytological analysis of cervical lesions. All 4043 women were either tested for HIV or had previously tested HIV positive. At baseline, 696 out of 4043 women (17.2\%) tested HR HPV positive by HC2. The cytology showed that overall $3.4 \% \quad(\mathrm{n}=139 / 4043)$ had HSIL+, while $8.1 \%(n=329 / 4043)$ of women had LSIL. A total 3416 women had both HPV- and cytology results. Among this subgroup of women, $18.9 \%$ were HPV-positive $(\mathrm{n}=644 / 3416)$, and the four most common HR HPV types were HPV 52 (3.8\%), HPV 16 (3.6\%), HPV 58 (2.5\%) and HPV 18 (2.4\%). Among HIV positive women, 33.7\% were HR HPV positive, while the corresponding figure among HIV-negative women was $15.6 \%$. Among women with high-grade lesions (HSIL+), 32.5\% had HPV 16, $19.3 \%$ had HPV 58, 17.5\% had HPV 31, 16.7\% had HPV 18 and $16.7 \%$ had HPV 52. A full description of the HPV distributions according to HIV status and cytology results is published elsewhere. ${ }^{33}$

\section{First follow-up findings}

A total of 3805 women (94\%) were eligible for first follow-up-238 women (6\%) were ineligible due to becoming pregnant, having moved, having had a hysterectomy or having become sick or died (figure 1). Of the 3805 women, 3074 women $(81 \%)$ attended the first follow-up visit approximately 14th months after enrolment.

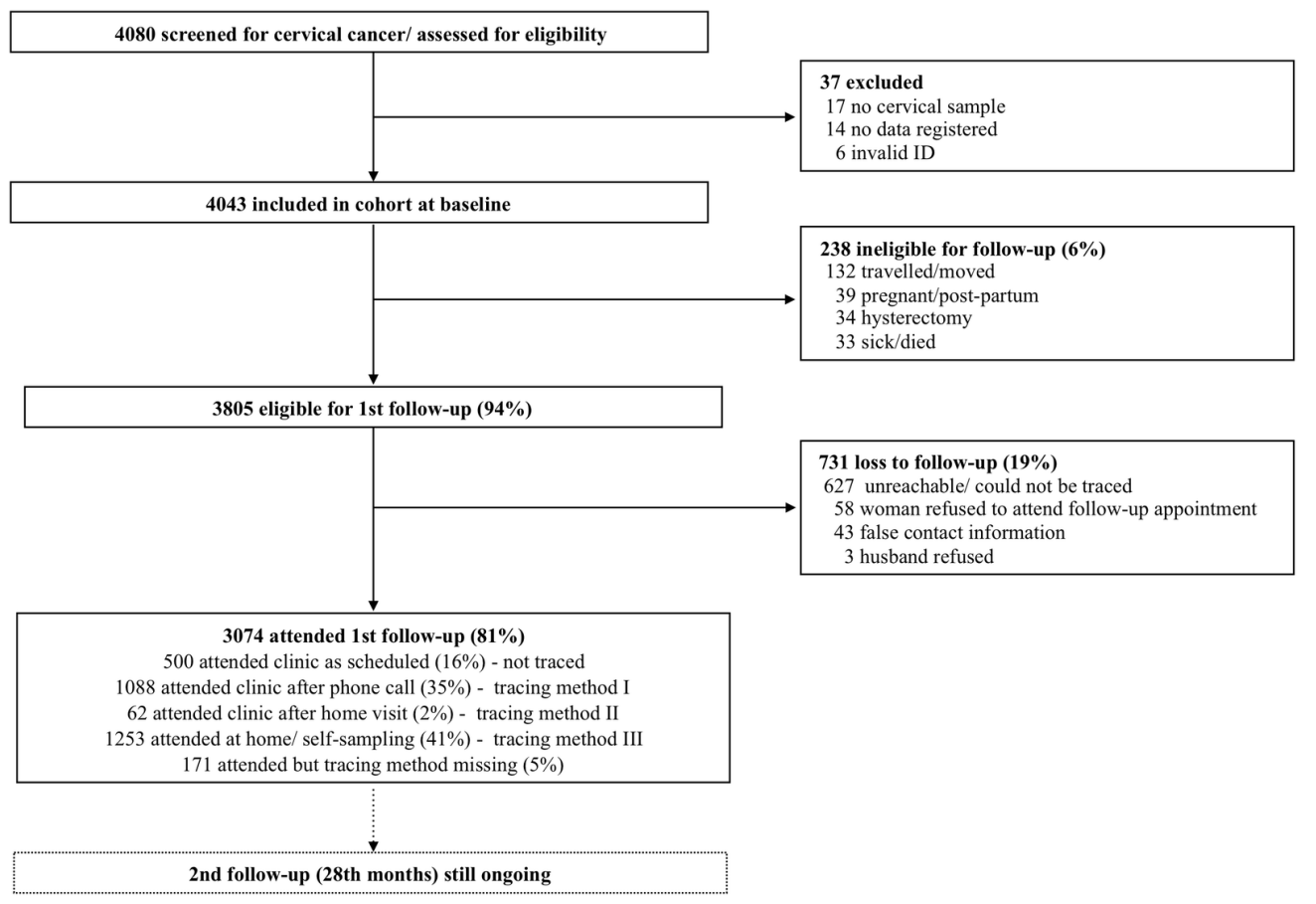

Figure 1 Flowchart of enrolment and follow-up of CONCEPT cohort. 
* จे

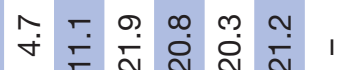

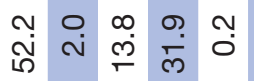

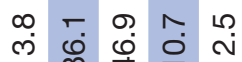

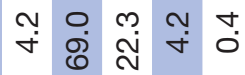

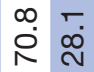

윽 ญำ ํำ

高 至

$z$

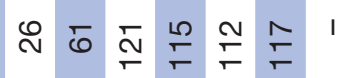

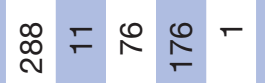

స 윰윰

$\stackrel{\sim}{\sim} \underset{\sim}{\sim} \stackrel{\sim}{\sim} \sim$

๙ ๑ ๙

논

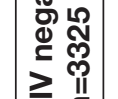

¿ $\stackrel{\text { III }}{\text { II }} \mathbf{z}$

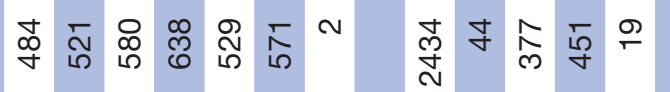

ஜ

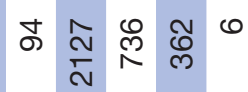

กัก 


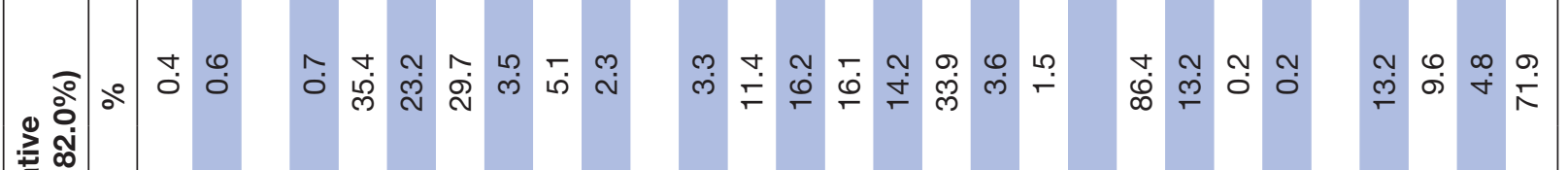

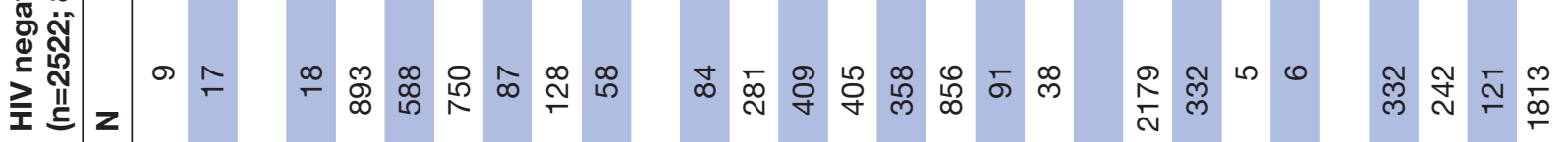

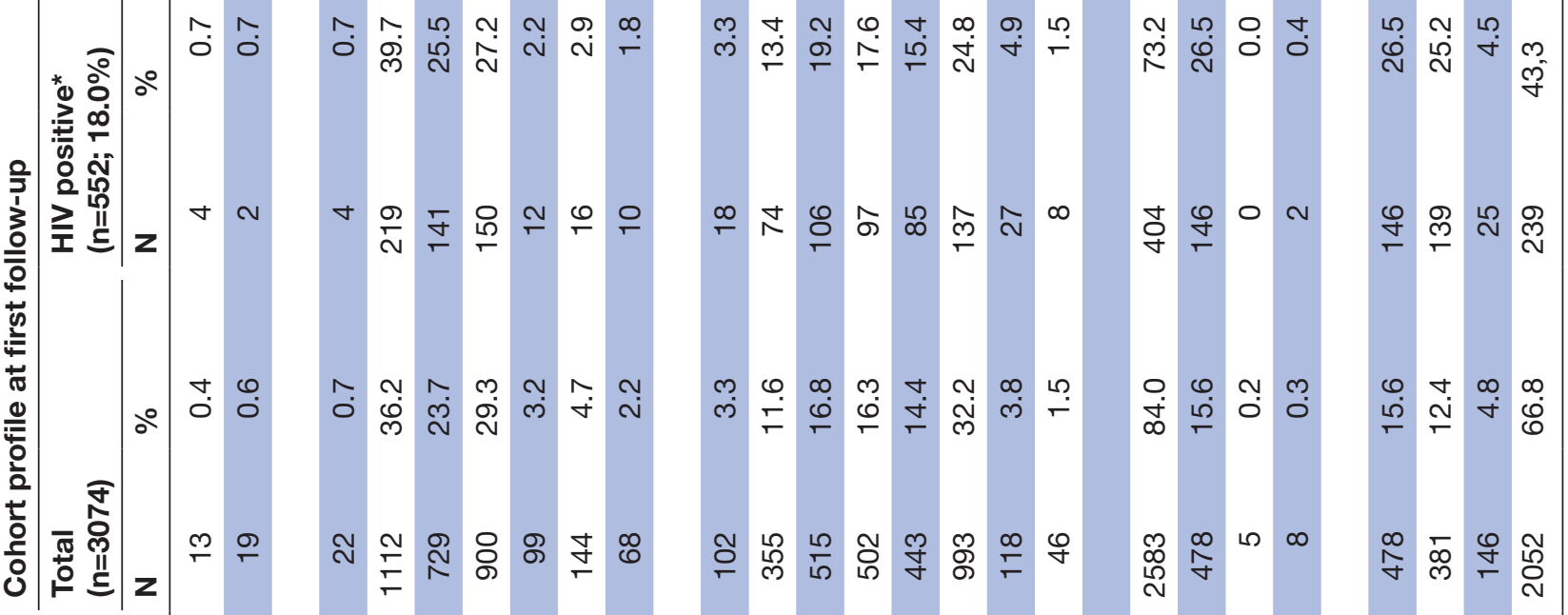

অ)

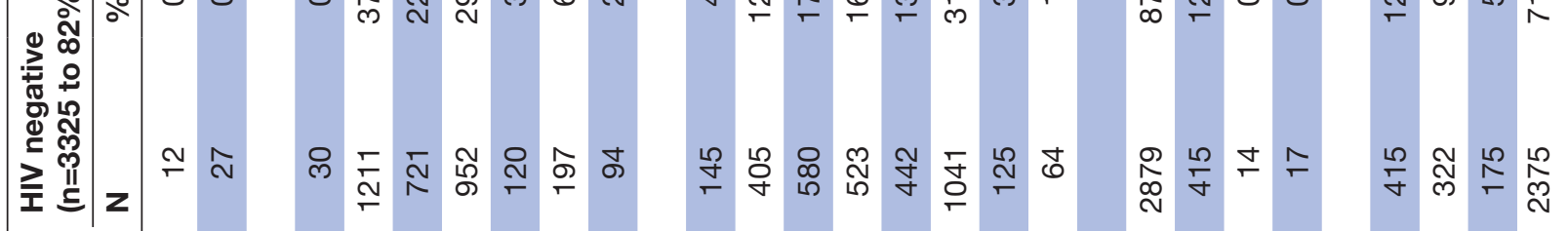

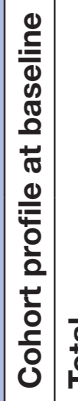

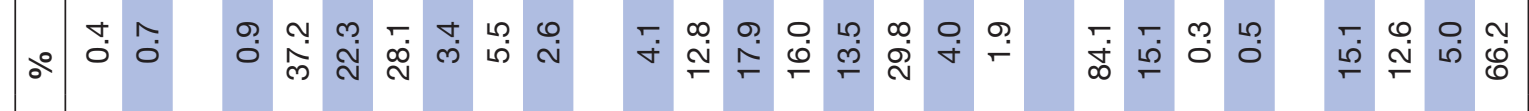

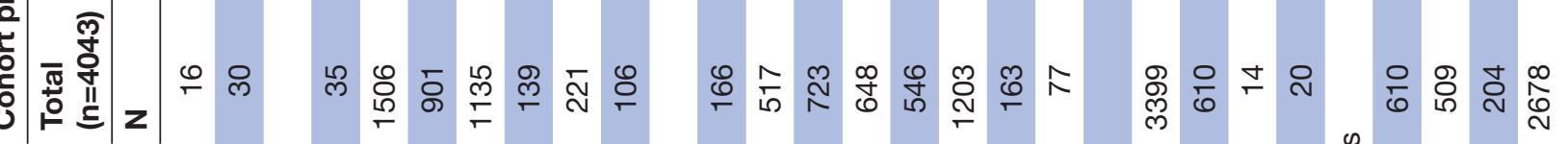

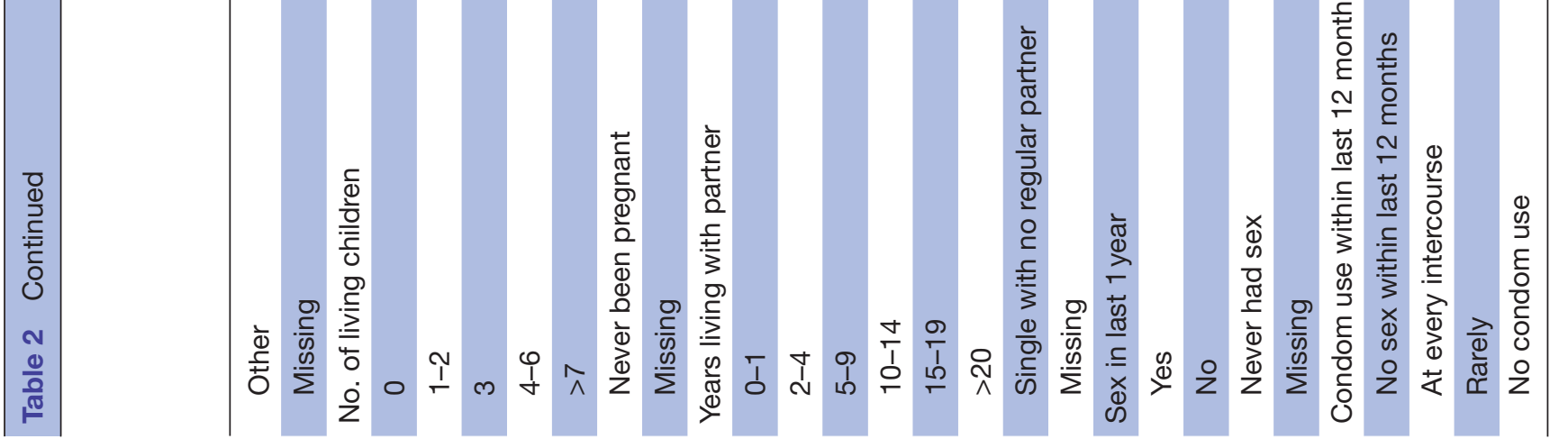




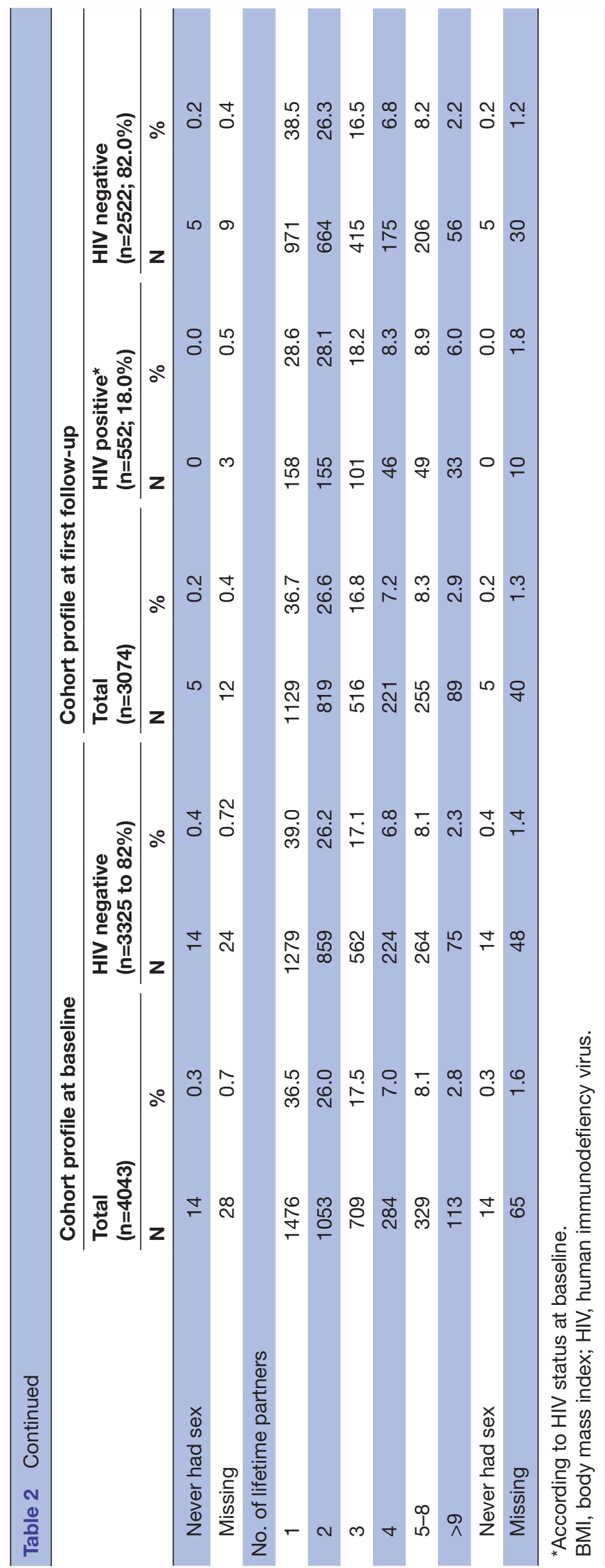


However, among the attendees, only 500 (16\%) attended within 30 days of their scheduled appointment date and without being traced for follow-up. A total of 1088 (35\%) attended the clinic after a phone call reminder (tracing method I), 62 women (2\%) attended the clinic after a nurse home-visit (tracing method II), while 1253 women $(41 \%)$ were followed up at home and had specimens collected using self-sampling device (tracing method III). A total of 731 women (19\%) were lost to follow-up (figure 1).

The women who participated in the first follow-up were very similar to those who did not attend when looking at sociodemographic factors (table 2). However, overall, fewer women were HR HPV positive at follow-up compared with baseline (14.8\% vs $17.2 \%)$, and fewer HIV positive women were HR HPV positive at follow-up compared with baseline (24.1\% vs $31.6 \%$ ) (table 3 ).

\section{STRENGTHS AND LIMITATIONS}

This is a large-scale longitudinal cervical cancer study conducted in an HIV endemic population that aims to address a major cause of disease among East-African women, which so far has not received much focus within global health research. Women are followed up over a long duration of time and with a large amount of data being collected using questionnaires and laboratory tests. Given the nature of our study, a significant attrition rate was expected. However, with the carefully designed tracing plans and dedicated home-visiting staff, we managed to attain an $81 \%$ participation rate at first follow-up. As women were enrolled during a patient-initiated screening at screening clinics, detailed HIV documentation was challenging to obtain as CTC cards were poorly documented or had not been brought to the screening. Despite the nurses calling these women after enrolment to retrieve the information, it was not provided by many HIV positive participants. This has led to a certain amount of missing values for a few variables and have limited our ability and power in analyses involving HIV immunologic markers and treatment. The HPV distribution found in this study population in comparison to data from the source population shows that the distribution is somewhat comparable, although it also differs to a high extent on some accounts. We found that among women with HSIL+, our study population had a higher prevalence of HPV 16 (32.5\% vs $30.2 \%)$ and HPV 58 (19.3\% vs $6.3 \%)$, a lower prevalence of HPV 52 (16.7\% vs $21.9 \%)$, while the prevalence of HPV 18 was the same $(16.7 \%) .{ }^{17}$ However, the data on the source population are based on one study conducted on ORCI in Dar es Salaam in $2014^{10}$; hence, the difference in the HPV distribution does not necessarily suggest that our study population is not generalisable to the source population but rather that it builds a stronger basis for understanding the true HPV distribution in Tanzania.

\section{FUTURE PLANS}

A second follow-up is underway (17 December 2018primo October 2020). Based on our large-scale data of both HPV infection in general and type-specific characterised by the women's HIV status, we plan to integrate these data with a previous cross-sectional HPV study (PROTECT study) conducted in this population as this can increase power in our findings. As we have already established a large cohort of participants, we foresee a potential to further characterise the HPV burden and establish risk factors over a longer course of time. Specifically, we wish to compare the clinical performance of three potential cervical cancer screening strategies in Tanzania, namely, (1) HC2 testing at varying cut-off points of viral load as

Table 3 HR HPV, HIV and cytology results at baseline and first follow-up

\begin{tabular}{|c|c|c|c|c|c|c|}
\hline \multicolumn{4}{|l|}{ Baseline } & \multicolumn{3}{|c|}{ First follow-up } \\
\hline \multicolumn{4}{|c|}{ Total $(n=4043)$} & \multicolumn{3}{|c|}{ Total $(n=3074)$} \\
\hline HPV & $\mathrm{n}$ & $\%$ & (95\% Cl) & $n$ & $\%$ & (95\% Cl) \\
\hline Negative & 2951 & 73 & (0.72 to 0.74$)$ & 2595 & 84.4 & (0.83 to 0.86$)$ \\
\hline Missing & 396 & 9.8 & (0.09 to 0.1$)$ & 41 & 1.3 & (0.01 to 0.02$)$ \\
\hline Positive & 718 & 17.8 & (0.17 to 0.19$)$ & 552 & 18 & (0.17 to 0.2$)$ \\
\hline Negative & 3325 & 82.2 & (0.81 to 0.83 ) & 2522 & 82 & (0.80 to 0.83 ) \\
\hline \multicolumn{7}{|l|}{ Cytology } \\
\hline HSIL & 139 & 3.4 & (0.03 to 0.04$)$ & & & \\
\hline LSIL & 329 & 8.1 & (0.07 to 0.09$)$ & & & \\
\hline
\end{tabular}

HIV, human immunodeficiency virus; HPV, human papillomavirus; HR, high risk; HSIL, high-grade squamous intraepithelial lesion; LSIL, lowgrade squamous intraepithelial lesion. 
measured by the RLU value; (2) HC2 testing with VIA triage; and (3) HC2 testing with triage using HPV16/18 genotyping. Further, we also foresee the possibility of linking our evidence with other groups in this population including males, adolescents and pregnant women. This may provide additional information on the similarities of the epidemiological burden among these groups and delineate differences in the correlations of HPV and HPV-related disease across groups.

\section{COLLABORATION}

Researchers interested in collaboration in this discipline especially in East and Central Africa are welcomed. This may be in extracting data from the project or jointly requesting further investigation from the cohort.

\section{Author affiliations}

${ }^{1}$ Department of Obstetrics and Gynaecology, Kilimanjaro Christian Medical Centre, Moshi, United Republic of Tanzania

${ }^{2}$ Department of Clinical Research, University of Southern Denmark, Odense, Denmark

${ }^{3}$ Department of Obstetrics and Gynaecology, Odense University Hospital, Odense, Denmark

${ }^{4}$ Department for Cancer Prevention Services, Ocean Road Cancer Institute, Dar es Salaam, United Republic of Tanzania

${ }^{5}$ Department of Public Health, Kilimanjaro Chrstian Medical College, Moshi, Tanzania ${ }^{6}$ Department of Virus, Lifestyle and Genes, Danish Cancer Society Research Center, Copenhagen, Denmark

${ }^{7}$ Department of Gynaecology, Copenhagen University Hospital, Copenhagen, Denmark

\section{Twitter Ditte Søndergaard Linde @ditte_linde}

Acknowledgements We would like to thank Danida for supporting this study and thank all partners involved in the CONCEPT study: screening nurses, outreach nurses, laboratory personnel and all the women who are part of the CONCEPT study.

Contributors JDM, RM, VR and SK designed the CONCEPT study. JDM, RM, VR, SK, JK, PS, CK, BM and DSL were involved in collecting data for the study. CW, SK, VR, BM and DSL were involved in data analysis and interpretation. CW, BM and DSL contributed to the conception of this article, and CW, BM, DSL, PS, JK, CK, VR, JDM, SK and RM were involved in the manuscript writing and revision. All authors read and approved the final manuscript.

Funding The work was supported by the Danish International Development Agency (Danida; 14-P02-Tan/A26775). The recipient of the grant was the primary investigator of the CONCEPT study (JM). The funders had no role in study design, data collection and analysis, decision to publish or preparation of the manuscript.

\section{Competing interests None declared.}

Patient and public involvement Patients and/or the public were not involved in the design, or conduct, or reporting, or dissemination plans of this research.

\section{Patient consent for publication Not required.}

Ethics approval The CONCEPT study was approved by the Ethical Committee of the Tanzanian National Institute of Medical Research (NIMR/HQ/R.8a/Vol.IX/1955) and is reported according to the Strengthening the Reporting of Observational studies in Epidemiology (STROBE) statement (online supplementary appendix 2).

Provenance and peer review Not commissioned; externally peer reviewed.

Data availability statement Data collected for the CONCEPT cohort study are available upon request. Individual participant data will de-identified. Additional available data include the CONCEPT eligibility and informed consent form, the CONCEPT contact information form, the protocol for how to deliver HPV-positive results to participants, the protocol for how to trace non-attendants. Data will be made available upon request by contacting the first or last author of this study by email at barikimchome@gmail.com/ dsondergaard@health.sdu.dk, who will then seek approval by the primary investigator in the CONCEPT project, Julius D. Mwaiselage.

Open access This is an open access article distributed in accordance with the Creative Commons Attribution Non Commercial (CC BY-NC 4.0) license, which permits others to distribute, remix, adapt, build upon this work non-commercially, and license their derivative works on different terms, provided the original work is properly cited, appropriate credit is given, any changes made indicated, and the use is non-commercial. See: http://creativecommons.org/licenses/by-nc/4.0/.

ORCID iD

Ditte Søndergaard Linde http://orcid.org/0000-0002-0851-6760

\section{REFERENCES}

1 Organization $\mathrm{WH}$. Improving data for decision-making: a toolkit for cervical cancer prevention and control programmes, 2018. Available: http://apps.who.int/iris

2 Serrano B, Brotons M, Bosch FX, et al. Epidemiology and burden of HPV-related disease. Best Pract Res Clin Obstet Gynaecol 2018;47:14-26.

3 Torre LA, Islami F, Siegel RL, et al. Global cancer in women: burden and trends. Cancer Epidemiol Biomarkers Prev 2017;26:444-57.

4 Bruni LB-RL AG, Serrano B. Human papillomavirus and related diseases in Tanzania.Summary report. 15. ICO Information Centre on HPV and Cancer, 2016.

5 Fokom-Domgue J, Combescure C, Fokom-Defo V, et al. Performance of alternative strategies for primary cervical cancer screening in subSaharan Africa: systematic review and meta-analysis of diagnostic test accuracy studies. BMJ 2015;351:h3084.

6 Bruni L, Diaz M, Castellsagué X, et al. Cervical human papillomavirus prevalence in 5 continents: meta-analysis of 1 million women with normal cytological findings. J Infect Dis 2010;202:1789-99.

7 Nowak RG, Gravitt PE, Morrison CS, et al. Increases in human papillomavirus detection during early HIV infection among women in Zimbabwe. J Infect Dis 2011;203:1182-91.

8 Dames DN, Blackman E, Butler R, et al. High-Risk cervical human papillomavirus infections among human immunodeficiency viruspositive women in the Bahamas. PLoS One 2014;9:e85429.

9 Looker KJ, Rönn MM, Brock PM, et al. Evidence of synergistic relationships between HIV and human papillomavirus (HPV): systematic reviews and meta-analyses of longitudinal studies of HPV acquisition and clearance by HIV status, and of HIV acquisition by HPV status. J Int AIDS Soc 2018;21:e25110.

10 Dartell MA, Rasch V, Iftner T, et al. Performance of visual inspection with acetic acid and human papillomavirus testing for detection of high-grade cervical lesions in HIV positive and HIV negative Tanzanian women. Int J Cancer 2014;135:896-904.

11 Linde DS, Korsholm M, Katanga J, et al. One-Way SMS and healthcare outcomes in Africa: systematic review of randomised trials with meta-analysis. PLoS One 2019;14:e0217485.

12 Akarolo-Anthony SN, Famooto AO, Dareng EO, et al. Age-Specific prevalence of human papilloma virus infection among Nigerian women. BMC Public Health 2014;14:656.

13 Guan P, Howell-Jones R, Li N, et al. Human papillomavirus types in 115,789 HPV-positive women: a meta-analysis from cervical infection to cancer. Int J Cancer 2012;131:2349-59.

14 Edna Omar V, Orvalho A, Nália I, et al. Human papillomavirus prevalence and genotype distribution among young women and men in Maputo City, Mozambique. BMJ Open 2017;7:e015653.

15 Husain RSA, Ramakrishnan V. Global variation of human papillomavirus genotypes and selected genes involved in cervical malignancies. Ann Glob Health 2015;81:675-83.

16 Ogembo RK, Gona PN, Seymour AJ, et al. Prevalence of human papillomavirus genotypes among African women with normal cervical cytology and neoplasia: a systematic review and meta-analysis. PLoS One 2015;10:e0122488.

17 Bruni LAG, Serrano B, Mena M, et al. ICO/IARC information centre on HPV and cancer (HPV information centre). human papillomavirus and related diseases in Tanzania. summary report 17 June, 2019.

18 Organization WH. Human papillomavirus (HPV) and cervical cancer, 2019. Available: https://www.who.int/en/news-room/fact-sheets/ detail/human-papillomavirus-(hpv)-and-cervical-cancer.

19 Bosch FX, Muñoz N. The viral etiology of cervical cancer. Virus Res 2002;89:183-90.

20 Kelly HA, Ngou J, Chikandiwa A, et al. Associations of human papillomavirus (HPV) genotypes with high-grade cervical neoplasia (CIN2+) in a cohort of women living with HIV in Burkina Faso and South Africa. PLoS One 2017;12:e0174117. 
21 Adebamowo SN, Dareng EO, Famooto AO, et al. Cohort profile: African collaborative center for microbiome and genomics research's (ACCME's) human papillomavirus (HPV) and cervical cancer study. Int J Epidemiol 2017:46:1745-1745j.

22 Dartell M, Rasch V, Kahesa C, et al. Human papillomavirus prevalence and type distribution in 3603 HIV-positive and HIVnegative women in the general population of Tanzania: the protect study. Sex Transm Dis 2012;39:201-8.

23 Watson-Jones D, Baisley K, Brown J, et al. High prevalence and incidence of human papillomavirus in a cohort of healthy young African female subjects. Sex Transm Infect 2013;89:358-65.

24 Houlihan CF, Baisley K, Bravo IG, et al. Rapid acquisition of HPV around the time of sexual debut in adolescent girls in Tanzania. Int $J$ Epidemiol 2016;45:762-73.

25 Baisley KJ, Andreasen A, Irani J, et al. Hpv prevalence around the time of sexual debut in adolescent girls in Tanzania. Sex Transm Infect 2020;96:211-9.

26 Koliopoulos G, Nyaga VN, Santesso N, et al. Cytology versus HPV testing for cervical cancer screening in the general population. Cochrane Database Syst Rev 2017;8:Cd008587.

27 Linde DS, Andersen MS, Mwaiselage JD, et al. Text messages to increase attendance to follow-up cervical cancer screening appointments among HPV-positive Tanzanian women (Connected2Care): study protocol for a randomised controlled trial. Trials 2017;18:555.

28 Linde DS, Rasch V, Mwaiselage JD, et al. Competing needs: a qualitative study of cervical cancer screening attendance among HPV-positive women in Tanzania. BMJ Open 2019;9:e024011.

29 Linde D, Andersen MS, Mwaiselage JD, et al. One-Way text messages versus no text messages on attendance to follow-up cervical cancer screening among HPV-positive Tanzanian women (Connected2Care): a parallel-group randomised controlled trial. SSRN Journal 2019:15863.
30 Bakiewicz A, Rasch V, Mwaiselage J, et al. "The best thing is that you are doing it for yourself" - perspectives on acceptability and feasibility of HPV self-sampling among cervical cancer screening clients in Tanzania: a qualitative pilot study. BMC Womens Health 2020;20:65

31 Katanga J, Kjaer SK, Manongi R, et al. Performance of careHPV, hybrid capture 2 and visual inspection with acetic acid for detection of high-grade cervical lesion in Tanzania: a cross-sectional study. PLoS One 2019;14:e0218559.

32 Linde DS, Andersen MS, Mwaiselage J, et al. Effectiveness of oneway text messaging on attendance to follow-up cervical cancer screening among human papillomavirus-positive Tanzanian women (Connected2Care): parallel-group randomized controlled trial. J Med Internet Res 2020;22:e15863.

33 Mchome BL, Kjaer SK, Manongi R, et al. Hpv types, cervical highgrade lesions and risk factors for oncogenic human papillomavirus infection among 3416 Tanzanian women. Sex Transm Infect 2020:054263.

34 jhpiego. Cervical cancer prevention guidelines for low-resource settings, 2011. Available: http://resources.jhpiego.org/system/files/ resources/cecap trainer 0. pdf

35 Dartell M, Rasch V, Munk C, et al. Risk factors for high-risk human papillomavirus detection among HIV-negative and HIV-positive women from Tanzania. Sex Transm Dis 2013;40:737-43.

36 Paul D, Blumenthal NM. Cervical cancer prevention guidelines for low-resource settings 2007: Jhpiego, 2005. Available: http:// reprolineplus.org/system/files/resources/CeCaPfullmanual.pdf

37 Nayar R, Wilbur DC. The Pap Test and Bethesda 2014. "The reports of my demise have been greatly exaggerated." (after a quotation from Mark Twain). Acta Cytol 2015;59:121-32.

38 Xu L, Padalko E, Oštrbenk A, et al. Clinical Evaluation of INNO-LiPA HPV Genotyping EXTRA II Assay Using the VALGENT Framework. Int J Mol Sci 2018;19:19092704. 